\title{
Flux pinning enhancement in ferromagnetic and superconducting thin-film multilayers
}

\author{
D. B. Jan, J. Y. Coulter, M. E. Hawley, L. N. Bulaevskii, M. P. Maley, and Q. X. Jiaa) \\ Superconductivity Technology Center, Los Alamos National Laboratory, Los Alamos, New Mexico 87545 \\ B. B. Maranville and F. Hellman \\ Department of Physics, University of California, San Diego, California 92093 \\ X. Q. Pan \\ Department of Materials Science and Engineering, University of Michigan, Ann Arbor, Michigan 48109
}

(Received 20 August 2002; accepted 9 December 2002)

\begin{abstract}
Flux pinning in high-temperature superconductors such as $\mathrm{YBa}_{2} \mathrm{Cu}_{3} \mathrm{O}_{7-x}(\mathrm{YBCO})$ in the past has been accomplished by pinning the vortex cores. We demonstrate magnetic-domain-induced flux pinning of the magnetic flux of vortices in a ferromagnet-superconductor bilayer consisting of CoPt grown on YBCO, where the ferromagnet has uniaxial perpendicular magnetic anisotropy and a random domain structure. We observe an improvement of the critical current due to magnetic pinning at temperatures close to the transition temperature. (C) 2003 American Institute of Physics.
\end{abstract}

[DOI: $10.1063 / 1.1542674]$

For practical applications, high temperature superconductors (HTS) such as $\mathrm{YBa}_{2} \mathrm{Cu}_{3} \mathrm{O}_{7-\delta}$ (YBCO) need to possess a high critical current density $\left(J_{c}\right)$. Enhancement of $J_{c}$ occurs by increasing the flux pinning capability of the superconductor. This is especially important in HTS, since thermal depinning readily occurs at liquid nitrogen temperature, in light of the small size of the vortex cores $(\sim 3 \mathrm{~nm}){ }^{1}$

Although flux pinning occurs naturally in YBCO due to crystallographic defects, flux pinning may be significantly enhanced by engineering microscopic defects that suppress superconductivity locally. It is energetically favorable for the normal core of a vortex to reside on nonsuperconducting regions within a superconductor. The maximum pinning energy per unit length in this case is the superconducting condensation energy in the volume of the vortex core, $U_{c p}$ $\sim\left(H_{c}^{2} / 8 \pi\right) \pi \xi^{2}=\left[\Phi_{0} / 8 \pi \lambda(T)\right]^{2}$, where $H_{c}$ is the thermodynamic critical field, $\xi$ is the coherence length (size of the normal core), $\Phi_{0}$ is the flux quantum, and $\lambda(T)$ is the temperature dependent London penetration depth $[\lambda(0)$ $\sim 150 \mathrm{~nm}$ for YBCO]. This pinning energy drops as $T \rightarrow T_{c}$ (critical temperature) due to the increase of $\lambda$ as $\lambda^{2}(0) / \lambda^{2}(T) \sim\left(1-T / T_{c}\right)$. Some flux pinning schemes include second phase inclusions, ${ }^{2}$ thickness modulation of the superconducting film, ${ }^{3}$ cold work-induced dislocations and strain, ${ }^{4}$ magnetic particles ${ }^{5}$ and $\operatorname{dots}^{6}$ on the superconductor surface, and columnar defects by ion irradiation. ${ }^{7-9}$

Recently, a method of flux pinning by magnetic domain walls was proposed by Bulaevskii et al. ${ }^{10}$ They suggest that stronger flux pinning of a single vortex (up to two orders of magnitude improvement versus pinning by columnar defects) might be realized by pinning the magnetic flux of the vortex, rather than just the core. In this scheme, the structure would be comprised of a ferromagnet-superconductor (FMSC) multilayer. The flux pinning would be provided by a ferromagnetic material with uniaxial perpendicular magnetic

${ }^{a)}$ Electronic mail: qxjia@lanl.gov anisotropy, such as $\mathrm{TbFe}$ or CoPt. They predicted that below the coercive field of the ferromagnetic film, the vortices should become trapped at domain boundaries, thereby providing flux pinning. It is estimated that the pinning barrier per unit length of a single vortex line is

$$
U_{m p} \sim \Phi_{0} M_{0},
$$

where $M_{0}$ is the magnetization of the ferromagnetic domain. This pinning energy is relevant for a vortex crossing a domain wall with the current normal to the domain wall in a stripe domain structure, but the pinning barrier is absent when vortices can move parallel to the domain walls. This pinning energy is almost temperature independent if the domain width $l>\lambda(T)$. This magnetic pinning energy supports a maximum current density $J_{c} \sim c M_{0} / l \sim 10^{7} \mathrm{~A} / \mathrm{cm}^{2}$ for $M_{0}$ $=100 \mathrm{emu} / \mathrm{cm}^{3}$ and $l=1000 \mathrm{~nm}$.

There have been several attempts at producing ferromagnet-superconductor bilayers to show the effects of flux pinning by using the above scheme. Garc a-Santiago, et al. ${ }^{11}$ produced an epitaxial $\mathrm{FM}-\mathrm{SC}$ bilayer consisting of $\mathrm{YBCO} / \mathrm{ZrO}_{2}-\mathrm{Y}_{2} \mathrm{O}_{3} \quad$ buffer $/ \mathrm{BaFe}_{12} \mathrm{O}_{19} / \mathrm{YSZ}(100)$. They found an upward shift in the irreversibility line compared to pure $\mathrm{YBCO}$, and they interpreted this as evidence of enhanced flux pinning. Zhang et al. ${ }^{12}$ have produced an epitaxial bilayer of $\mathrm{YBCO} / \mathrm{Pr}_{0.67} \mathrm{Sr}_{0.33} \mathrm{MnO}_{3}(\mathrm{PSMO}) / \mathrm{SrTiO}_{3}$. From their magnetization measurements, they indicated that the in-plane magnetized PSMO somehow also enhances flux pinning. Unlike the aforementioned reports, the results presented in this letter show an enhancement in flux pinning by transport measurements in magnetic field in a bilayer structure. The improvement in critical current density $J_{c} \sim 2-3$ times provides the most direct evidence of flux pinning. Also, the experimental results agree qualitatively with the theoretical expectation from Bulaevskii et al. ${ }^{10}$

YBCO films were grown by pulsed laser deposition on pseudocubic (100) oriented $\mathrm{LaAlO}_{3}$ (LAO) substrates obtained from Lucent Technologies. Ablation was performed 


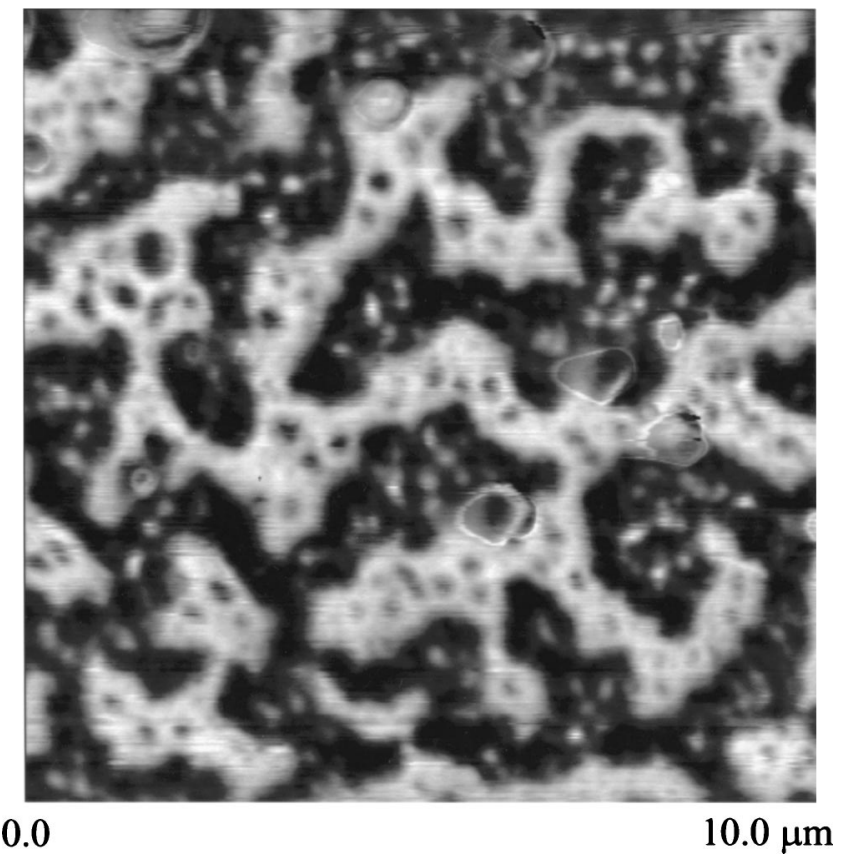

FIG. 1. MFM image of a multilayer CoPt on YBCO, showing maze-like domain structure with uniaxial perpendicular magnetic anisotropy.

using a XeCl excimer laser (energy density $=2 \mathrm{~J} / \mathrm{cm}^{2}$, targetsubstrate distance $=4.5 \mathrm{~cm}$ ). All of our YBCO depositions were carried out with the substrate at $780^{\circ} \mathrm{C}$ and pure $\mathrm{O}_{2}$ pressure at 200 mTorr. Following the deposition, the YBCO films were naturally cooled in 250 Torr $\mathrm{O}_{2}$. Our processing conditions yielded epitaxial $c$-axis oriented YBCO, where the $\mathrm{CuO}$ planes lie parallel with the substrate.

Samples were prepared for electrical property measurements using photolithography to produce microbridge patterns with dimensions $2.0 \mathrm{~mm}$ in length by $250 \mu \mathrm{m}$ wide. $\mathrm{Ag}$ contacts were applied to the YBCO film surface by thermal evaporation, and the films subsequently were annealed at $550^{\circ} \mathrm{C}$ for $30 \mathrm{~min}$ in flowing oxygen to minimize contact resistance. For all of our $J_{c}$ measurements, we used the standard $1 \mu \mathrm{V} / \mathrm{cm}$ criterion.

Multilayer films with a thickness of $200 \mathrm{~nm} \mathrm{CoPt}$ were grown directly on top of the YBCO film by sputtering $0.9 \mathrm{~nm}$ $\mathrm{Pt} / 0.2 \mathrm{~nm}$ Co repeats at room temperature in high vacuum $\left(<2 \times 10^{-8}\right.$ Torr), with $1.5 \mathrm{~nm}$ Pt capping layers at both the beginning and end of the deposition to prevent Co oxidation. Although a higher deposition temperature would have yielded higher coercivity and magnetization saturation values for the CoPt ${ }^{13}$ we maintained the growth temperature at room temperature to ensure that the $\mathrm{YBCO}$ would not lose its superconducting properties by diffusion-induced loss of oxygen.

Figure 1 shows a magnetic force microscopy (MFM) image of the $200 \mathrm{~nm} \mathrm{CoPt}$ multilayer on $500 \mathrm{~nm}$ YBCO. Immediately noticeable in the MFM image are the maze-like domain structures in the CoPt multilayer, which have a fairly uniform domain width on the order of $1.0 \mu \mathrm{m}$. An interesting feature of these films is the finer domain structure filling in the areas between the major domain structures. Similar MFM images were obtained for $200 \mathrm{~nm}$ CoPt directly on LAO, graphite, and glass substrate (not shown). These irregular magnetic domains are similar to those of TbFe. ${ }^{13}$ It should

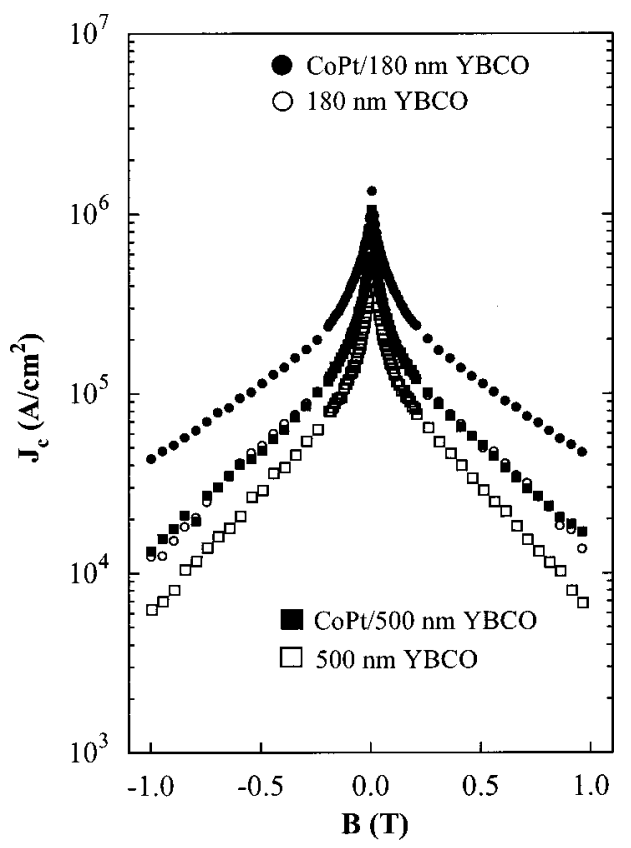

FIG. 2. $J_{c}$ vs $B \|_{c}$ transport measurements for 500 and $180 \mathrm{~nm}$ YBCO films at $86 \mathrm{~K}$ with and without CoPt multilayer applied.

also be noted that the CoPt multilayer showed strong perpendicular anisotropy, as can be seen from the sharpness of the domain boundaries and the high contrast between domains of opposite polarity. The MFM scans, which were taken in the frequency mode using a Digital Instrument Nanoscope IIIA multimode probe, showed strong contrast, further indicating strong magnetic anisotropy. The origin of magnetic perpendicular anisotropy in these CoPt multilayers is believed to be due a magnetoelastic effect resulting from the $8 \%$ lattice mismatch between Co and Pt. ${ }^{14,15}$ We also measured the coercivity and magnetic saturation in the hard in-plane direction and easy $c$ axis using the dc superconducting quantum interference device (SQUID) magnetometer at $75 \mathrm{~K}$. In this measurement, we fixed the orientation of the films in the sample holder with respect to the known orientation of the magnetic field within the SQUID. As expected, the film has uniaxial magnetic perpendicular anisotropy with a higher coercivity $H_{\text {coer }}(B \| a b) \sim 2500 \mathrm{G}$ and magnetic saturation $M_{s}(B \| a b) \sim 210 \mathrm{emu} / \mathrm{cm}^{3}$ in the hard in-plane direction than in the easy $c$ axis, with corresponding values $H_{\text {coer }}(B \| c)$ $\sim 600 \mathrm{G}$ and $M_{s}(B \| c) \sim 135 \mathrm{emu} / \mathrm{cm}^{3}$. From Eq. (1), such a domain structure with width $l \sim 1000 \mathrm{~nm}$ provides a maximum pinning energy per vortex line $\Phi_{0} M_{0} d_{s}=4 \times 10^{6} \mathrm{~K}$ ( $d_{s}$ is the YBCO film thickness) at temperatures below $\sim 0.99 T_{c}$, where $\lambda(T)<l$.

Improvement in flux pinning capability near $T_{c}$, is clearly demonstrated by comparing the transport measurements in a magnetic field $(B \| c)$ before and after adding 200 $\mathrm{nm}$ CoPt on both the 500 and $180 \mathrm{~nm}$ YBCO films. No significant improvement in $J_{c}$ was observed at $75 \mathrm{~K}$, indicating that at this temperature, defect induced pinning is still dominant. By contrast, Fig. 2 shows $J_{c}$ as a function of applied magnetic field $\left(B \|_{c}\right)$ before and after the CoPt coating is applied on the YBCO films, in liquid argon at $86 \mathrm{~K}$. We see from the data that in both the $500 \mathrm{~nm}$ YBCO and $180 \mathrm{~nm}$ YBCO samples, the change in $J_{c}$ by a factor of 2-3 upon addition of CoPt is present at $86 \mathrm{~K}$, which is closer to the 


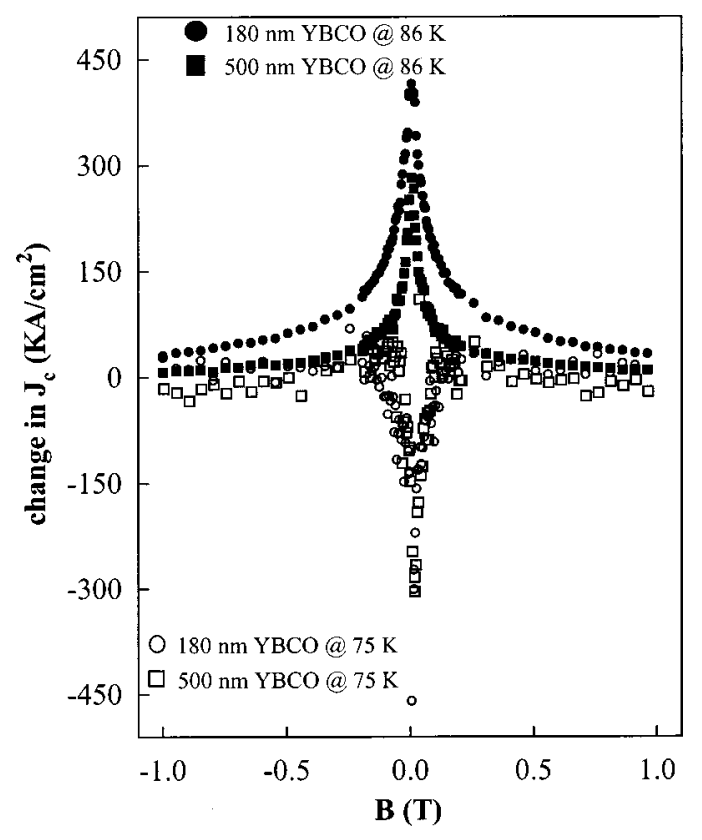

FIG. 3. Difference of $J_{c}$ (with and without CoPt layer applied) vs $B \|_{c}$ transport measurements for 500 and $180 \mathrm{~nm}$ YBCO films at 75 and $86 \mathrm{~K}$.

transition temperature. This observation is exactly what is expected from the theory. ${ }^{10}$ We should also note that we are improving the flux pinning capability of already very high quality YBCO. At $75 \mathrm{~K}$ in its self-field, the $J_{c}$ for the $500 \mathrm{~nm}$ YBCO sample is $4.28 \times 10^{6} \mathrm{~A} / \mathrm{cm}^{2}$ and $J_{c}$ for $180 \mathrm{~nm} \mathrm{YBCO}$ is $4.71 \times 10^{6} \mathrm{~A} / \mathrm{cm}^{2}$. At $86 \mathrm{~K}$ in self-field, the $J_{c}$ for the 500 $\mathrm{nm}$ YBCO sample is $7.91 \times 10^{5} \mathrm{~A} / \mathrm{cm}^{2}$ and $J_{c}$ for the $180 \mathrm{~nm}$ YBCO film is $9.82 \times 10^{5} \mathrm{~A} / \mathrm{cm}^{2}$. Figure 3 shows the change in $J_{c}$ due to the application of the CoPt multilayer on the 500 and $180 \mathrm{~nm}$ YBCO films at 75 and $86 \mathrm{~K}$. As can be seen from Fig. 3, at $86 \mathrm{~K}$ for both the 500 and $180 \mathrm{~nm}$ samples, the addition of CoPt increases $J_{c}$ both in the presence and absence of applied field $\left(B \|_{c}\right)$. In terms of absolute change, the boost in $J_{c}$ is most significant at moderate fields between approximately -600 and $600 \mathrm{G}$ with a maximum at $0 \mathrm{G}$, and it tails off at higher field.

Recall that the coercivity $H_{\text {coer }}(B \| c)$ along the easy direction of the CoPt film is $600 \mathrm{G}$. We can then understand the results at $86 \mathrm{~K}$ as follows. Below $-600 \mathrm{G}$ and above $600 \mathrm{G}$, the domains approach saturation along the direction of the applied field. Above $H_{\text {coer }}$ and below $-H_{\text {coer }}$, the ferromagnetic film appears to have little net effect on vortex pinning, as the contribution from flux pinning from the shrinking magnetic domains diminishes. Between $-H_{\text {coer }}$ and $H_{\text {coer }}$, while approaching zero field, the number of domains with up and down magnetizations are approaching equilibrium, and this provides maximum vortex magnetic flux pinning. Closer to zero field within $H_{\text {coer }}$, at $75 \mathrm{~K}$ flux pinning from natural pinning sites in $\mathrm{YBCO}$ is stronger than at $86 \mathrm{~K}$.

In comparing the flux pinning results between $500 \mathrm{~nm}$ YBCO and $180 \mathrm{~nm}$ YBCO superconductor layer, the changes upon addition of $200 \mathrm{~nm} \mathrm{CoPt}$ are more pronounced for the thinner $180 \mathrm{~nm}$ YBCO sample both at 75 and $86 \mathrm{~K}$. We may understand this as greater penetration of the magnetic field into the thinner YBCO film, thereby enhancing both the strength of flux pinning and intrinsic magnetic field the sample experiences. The magnitude of $J_{c}$ at $86 \mathrm{~K}$ is $\sim 10$ times smaller than predicted above for the maximum current densities supported by magnetic domain wall pinning. This is likely due to the random pattern of the stripe domains seen in these CoPt films (see Fig. 1), which facilitates movement of vortices along domain boundaries. To optimize pinning by this mechanism, the superconducting film can be placed between two ferromagnetic films with different domain patterns, assuming they do not influence each other.

In summary, flux pinning enhancement in a ferromagnetsuperconductor bilayer has been demonstrated using transport measurements. Flux pinning in YBCO by pinning entire vortices at magnetic domain boundaries with uniaxial perpendicular magnetic anisotropy has been shown to be effective at temperatures close to $T_{c}(\sim 86 \mathrm{~K})$ and within $\pm H_{\text {coer }}$. At this temperature, the magnetic domain pinning dominates defect induced pinning.

${ }^{1}$ M. N. Wilson, Superconducting Magnets (Oxford University Press, Oxford, 1983).

${ }^{2}$ C. Meingast and D. C. Larbalestier, J. Appl. Phys. 66, 5962 (1989).

${ }^{3}$ O. Daldini, P. Martinoli, J. L. Olsen, and G. Berner, Phys. Rev. Lett. 32, 218 (1974).

${ }^{4}$ J. W. Ekin, J. Appl. Phys. 62, 4829 (1987).

${ }^{5}$ Y. Otani, B. Pannetier, J. P. Nozieres, and D. Givord, J. Magn. Magn. Mater. 126, 622 (1993).

${ }^{6}$ J. I. Martin, M. Velez, J. Nogues, and I. K. Schuller, Phys. Rev. Lett. 79, 1929 (1997)

${ }^{7}$ N. Chikumoto, M. Konczykowski, T. Terai, and M. Murakami, Supercond. Sci. Technol. 13, 749 (2000).

${ }^{8}$ D. R. Nelson and V. M. Vinokur, Phys. Rev. Lett. 68, 2398 (1992).

${ }^{9}$ L. Civale, A. D. Marwick, T. K. Wortington, M. A. Kirk, J. R. Thompson, L. Krusin-Elbaum, Y. Sun, J. R. Clem, and F. Holzberg, Phys. Rev. Lett. 67, 648 (1991).

${ }^{10}$ L. N. Bulaevskii, E. M. Chudnovsky, and M. P. Maley, Appl. Phys. Lett. 76, 2594 (2000).

${ }^{11}$ A. Garc a-Santiago, F. Sanchez, M. Varela, and J. Tejada, Appl. Phys. Lett. 77, 2900 (2000).

${ }^{12}$ X. X. Zhang, G. H. Wen, R. K. Zheng, G. C. Xiong, and G. J. Lian, Europhys. Lett. 56, 119 (2001).

${ }^{13}$ F. Hellman, A. L. Shapiro, E. N. Abarra, R. A. Robinson, R. P. Hjelm, P. A. Seeger, J. J. Rhyne, and J. I. Suzuki, Phys. Rev. B 59, 11408 (1999).

${ }^{14}$ T. Kingetsu, J. Appl. Phys. 76, 4267 (1994).

${ }^{15}$ I. S. Pogosova, J. V. Harzer, B. Hillebrands, G. Gntherodt, D. Guggi, D. Weller, R. F. C. Farrow, and C. H. Lee, J. Appl. Phys. 76, 908 (1994). 